\title{
Identification of systems with unknown inputs using indirect input measurements
}

\author{
Jonas Linder and Martin Enqvist \\ Journal Article
}

\section{Tweet}

N.B.: When citing this work, cite the original article.

This is an electronic version of an article published in:

Jonas Linder and Martin Enqvist, Identification of systems with unknown inputs using indirect input measurements, International Journal of Control, 2017. 90(4), pp.729-745.

International Journal of Control is available online at informaworldTM:

http://dx.doi.org/10.1080/00207179.2016.1222557

Copyright: Taylor \& Francis: STM, Behavioural Science and Public Health Titles http://www.tandf.co.uk/journals/default.asp

Postprint available at: Linköping University Electronic Press

http://urn.kb.se/resolve?urn=urn:nbn:se:liu:diva-136198

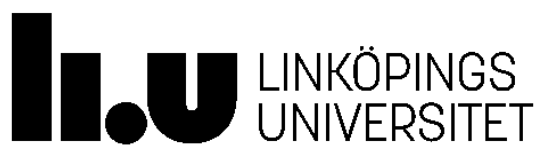


This is the author manuscript of

\section{Identification of systems with unknown inputs using indirect input measurements}

Jonas Linder and Martin Enqvist

To reference this work, please cite:

Jonas Linder and Martin Enqvist. Identification of systems with unknown inputs using indirect input measurements. International Journal of Control, 90(4):729-745, Feb 2017.

DOI: $10.1080 / 00207179.2016 .1222557$.

To link to this work, please use:

http://dx.doi.org/10.1080/00207179.2016.1222557 


\title{
Identification of Systems with Unknown Inputs Using Indirect Input Measurements
}

\author{
Jonas Linder* and Martin Enqvist \\ Division of Automatic Control at Department of Electrical Engineering, Linköping University, Sweden \\ (Received 29 January 2016; accepted 04 August 2016)
}

\begin{abstract}
A common issue with many system identification problems is that the true input to the system is unknown. This paper extends a previously presented indirect modeling framework that deals with identification of systems where the input is partially or fully unknown. In this framework, unknown inputs are eliminated by using additional measurements that directly or indirectly contain information about the unknown inputs. The resulting indirect predictor model is only dependent on known and measured signals and can be used to estimate the desired dynamics or properties. Since the input of the indirect model contains both known inputs and measurements that could all be correlated with the same disturbances as the output, estimation of the indirect model has similar challenges as a closed-loop estimation problem. In fact, due to the generality of the indirect modeling framework it unifies a number of already existing system identification problems that are contained as special cases. For completeness, the paper is concluded with one method that can be used to estimate the indirect model as well as an experimental verification to show the applicability of the framework.
\end{abstract}

Keywords: System identification, Model structure, Physical models, Instrumental variable, Closed-loop

\section{Introduction}

In many engineering applications there is an interest to study or control a system's behavior under some kind of unknown excitation. In robotics, for example, the forces acting on the manipulator might be unknown due to varying payload. In the automotive industry, there are unknown variations in gravitational forces due to the slope of the road, and in marine applications, the forces induced by wind and waves are typically unknown. Other examples include propagation of vibrations in structures, such as bridges or airplanes, where the forces inducing the vibrations are unknown and telecommunication where unknown data is sent through a channel with unknown properties.

The treatment of the unknown excitation varies. Sometimes, it is considered a disturbance and is either neglected or partially mitigated, for example, by using feedback control. Other situations might require a different approach, for instance, due to genuine interest in the unknown excitation, or a large impact of the unknown excitation which might lead to bad performance if it is ignored. Knowledge of the unknown excitation might thus be crucial. However, measuring the unknown signal might be impossible or impractical in many of the mentioned applications. One solution is to measure related signals and to use a model-based approach in order to compensate for the excitation signal or to reconstruct it. One way of obtaining models is to use system identification.

The objective of system identification is to estimate a model between an input and the resulting output using measured data. Common assumptions are that the relevant output signals are measurable and that the inputs are known. Under these assumptions, one important issue is to mitigate the effects of disturbances, present in the measured signals, such that an accurate model of the input-output behavior can be estimated. A classical example is 
the open-loop system identification problem, where the output is corrupted by an unknown disturbance while the input is assumed to be known and uncorrelated with the disturbance (Ljung, 1999). Slightly more complex problems arise when the input is still assumed to be known but is correlated with the same disturbance as the output. An example is the classical closed-loop system identification problem where the correlation occurs due to feedback, i.e. the input depends on the output, and possibly an external reference signal, through a controller (Forssell \& Ljung, 1999). A generalization of the closed-loop identification problem is dynamic network identification where a set of nodes (signals) are connected to each other through dynamic systems and the goal is to estimate an interesting subset of the dynamics. Disturbances are assumed to enter at each node and the correlation between disturbances acting on the signals used as inputs and the signals used as outputs are determined by the interconnection structure of the network (Dankers, 2014; Van den Hof, Dankers, Heuberger, \& Bombois, 2013).

In many system identification problems, it is assumed that the input is unknown. One example is problems where the input is unknown but is assumed to be measured with noise. A typical example is the errors-in-variables (EIV) problem where the emphasis lies in compensating for the effects of noise on the in- and output data (Söderström, 2007). Note that this is a fundamentally different problem to the closed-loop problem where the input disturbance actually enters the system through the input. However, closed-loop EIV problems have also been considered (Söderström, Wang, Pintelon, \& Schoukens, 2013) and in the general dynamic network identification setup, all signals are typically assumed to be measured with noise (Dankers, 2014).

Another class of unknown input problems is when assumptions are made on the input to the system. One example is time-series analysis where the input is assumed to be a stochastic process, for example, filtered white noise, and the goal is to estimate a model that can predict future outputs based on current and previous outputs only (Shumway \& Stoffer, 2010). Another example of unknown input problems with assumptions on the input is the related problem of blind identification (Abed-Meraim, Qiu, \& Hua, 1997) which had its origins in blind deconvolution (Stockham, Cannon, \& Ingebretsen, 1975). In blind identification, the input is not necessarily assumed to be stochastic and it is estimated in conjunction with a model of the system (Abed-Meraim et al., 1997).

A third class of unknown input problems is sensor-to-sensor system identification problems (S2SID). In the S2SID framework, several measurements are assumed to be dependent on the unknown input. Some measurements are used as output while other measurements are used as input to what is called a pseudo transfer function. Unfortunately, dynamics that is shared, in the transfer functions from the unknown input to the measurements, will cancel, but more importantly, so does the unknown input Aljanaideh, Ali, Holzel, Kukreja, \& Bernstein, 2012, D’Amato, Brzezinski, Holzel, Ni, \& Bernstein, 2009).

The idea of elimination of unknown inputs has also been approached in other fields. Unknown input observers (UIO), or disturbance decoupled observers, have been developed since the 1970's and have mainly been used in the field of fault detection. The UIO is an observer where the difference between the state estimate and the true state is independent of the unknown input. The idea of a disturbance-decoupled equivalent model where the unknown input had been eliminated from the model was introduced in Hou and Müller (1994) and extended in Hou and Patton (1998). The equivalent model description thus separates the step of eliminating the disturbance from the actual observer design which means that an arbitrary design scheme can be used to obtain the observer. 
In this paper, the modeling framework of Linder and Enqvist (2015) is extended to deal with additional input measurements and a deeper analysis of the framework is provided. The goal of the framework is to estimate a model of a subsystem $G_{O}$ which is part of a larger system, from the partially unknown input vector $u$ to some of the available measurements $y_{0}$. Two types of external signals are assumed to affect the system, user-controlled signals affecting the input, and unknown disturbances affecting both the input and output. It is assumed that all elements of the input vector possibly are correlated with the external usercontrolled signal and hence, neglecting some inputs could cause a bias in the remaining parts of the model estimator.

In the indirect modeling framework, the effects of the unknown inputs are assumed to be visible in several additional measurements that are divided into direct and indirect input measurements. The direct input measurements $y_{D}$ are similar to the input measurements in EIV problems and directly measure the unknown input $u_{D}$ with noise through known dynamics. In contrast, the unknown inputs $u_{I}$ are only measured through possibly unknown dynamics and the measurement may also be dependent on all inputs. The measurements thus indirectly contain information about the inputs and are called indirect input measurements. The unknown inputs are eliminated through algebraic manipulations and the resulting indirect model can be used in conjunction with the output, the input measurements and the known inputs, to estimate the dynamical model of interest. The algebraic manipulations are similar to those resulting in the disturbance-decoupled equivalent model of Hou and Patton (1998) but the equivalent model is formed from a known model and under stricter assumptions than in the indirect framework.

The resulting estimation problem shares the challenges of closed-loop system identification since measurements that are used as inputs are assumed to be correlated with the same disturbance as the output. In fact, due to the assumed generality of the framework, many of the classical and recently proposed system identification problems mentioned earlier are special cases of the proposed indirect modeling framework. Hence, the indirect modeling framework can be viewed as a unified approach to these problems and as a way to deal with combined problems, e.g. combined open- and closed-loop problems, S2SID problems with additional known inputs or dynamic networks with unknown inputs. For completeness, an iterative instrumental variable method based on the external user-controllable signals is proposed as one example of how to estimate the desired model and the applicability of the method is shown in an experimental study.

The outline of this paper is as follows: In Section 2 the problem is formulated. Section 3 presents the indirect framework: the process of eliminating the unknown inputs using input measurements, properties of the indirect model, input and output selection and connections between the indirect framework and existing system identification problems. In Section 4 , the possible benefits of using the indirect framework are discussed in terms of identifiability, consistency and variance properties. Section 5 presents the experimental verification and finally, in Section 6 the paper is concluded.

\section{Problem Formulation - Systems with Unknown Input}

Figure 1(a) shows the linear system $G$ with the output $y \in \mathbb{R}^{n_{y}}$ which is assumed to be influenced by two types of external signals, user-controllable signals $\delta \in \mathbb{R}^{n_{\delta}}$ and uncontrollable signals $\tau \in \mathbb{R}^{n_{\tau}}$ called disturbances. The system contains some unknown dynamics or properties that are desired to be estimated from data. Estimating a complete model of the 
system $G$ is assumed to be intractable, for instance, due to the complexity of the system or due to essential measurements not being available. Instead, only a subsystem $G_{O}$ of $G$, which contains the interesting dynamics or properties, is estimated using the model

$$
y_{O}=G_{O}(\mathrm{p}) u+\underbrace{H_{O}(\mathrm{p}) \tau}_{\tau_{O}}
$$

where $y_{O} \in \mathbb{R}^{n_{O}}$ is a subset of the available measurements $y, u \in \mathbb{R}^{n_{u}}$ is the input, $\mathrm{p}$ is the differential operator and $G_{O}(\mathrm{p})$ and $H_{O}(\mathrm{p})$ are proper transfer function matrices of suitable size, see Figure 1(b). Note that the input not necessarily is the actual physical input to the system. For example, in control applications it is common to consider the output of the controller generated by the computer to be the input to the system while the physical input, for instance, is a force generated by an actuator. The discrepancy between the input and the physical input thus has to be captured by the model. In the end, we are interested in a model between the signals defined as input and output.

Remark 1 (Continuous- or discrete-time modelling): Here, the models will be given in continuous time but discrete-time models can be used instead. The choice between continuousand discrete-time models is dependent on what best suits the application and the chosen estimation method. Using a continuous-time model might be beneficial, for instance, if the system is stiff or the data has varying sample time, see Garnier and Young (2014) for details.

The input $u$ is assumed to be related to the external signals $\delta$ and $\tau$ through the relation

$$
u=F_{\delta}(\mathrm{p}) \delta+F_{\tau}(\mathrm{p}) \tau
$$

where $F_{\delta}(\mathrm{p})$ and $F_{\tau}(\mathrm{p})$ are possibly unknown transfer function matrices of suitable dimensions. It is assumed that the input $u$ captures all contributions from the external usercontrollable signal $\delta$, i.e. that there are no paths from the external signal $\delta$ to the output $y_{O}$ not passing through the input $u$. Furthermore, the input $u$ is assumed to be partially unknown and is divided into

$$
\left.u=\left[\begin{array}{l}
u_{K} \\
-u_{I} \\
u_{D}
\end{array}\right]\right\} \text { known }
$$

where $u_{K} \in \mathbb{R}^{n_{K}}$ are known inputs, $u_{D} \in \mathbb{R}^{n_{D}}$ are directly measured inputs and $u_{I} \in \mathbb{R}^{n_{I}}$ in-
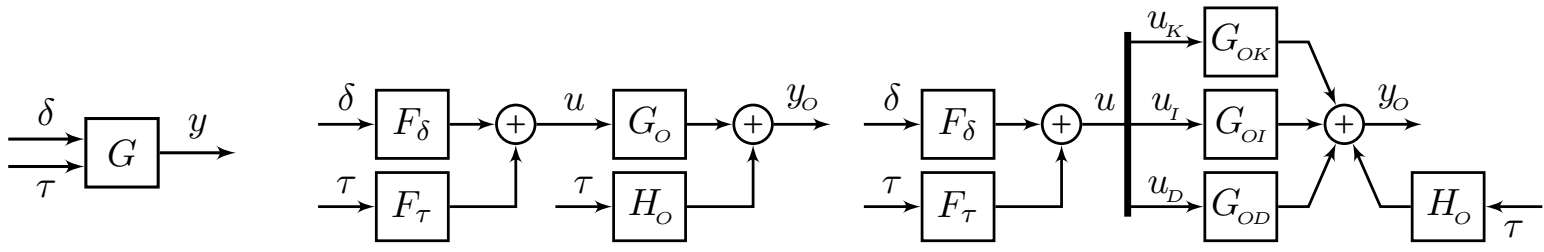

(a) The system $G$ with its (b) Equations $\sqrt{1}$ and 2 represented as output $y$ determined by the a block diagram showing the dependen-

(c) Equations 22 and (5) represented as a block diaexternal signals $\delta$ and $\tau$. cies on the external signals $\delta$ and $\tau$. gram showing the division of the input and the depen-

Figure 1. A system's output $y$ is affected by two types of external signals, $\delta$ and $\tau$, see (a) It is assumed that estimating a complete model of the system $G$ is intractable. Instead, a subsystem $G_{O}$, which contains the interesting dynamics or properties, with the output $y_{O}$ and the input $u$ is estimated, see (b) The input $u$ is divided according to (3) which results in 5, see (c) 
directly measured inputs. Let us for the moment assume that it is essential or at least beneficial to include all the unknown inputs in the modeling. The partitioning or classification of the unknown inputs and the necessity or benefits of including them will later be discussed Sections 3 and 4 .

To be consistent with the input partitioning, the transfer function matrix $G_{O}(\mathrm{p})$ is divided into

$$
G_{O}(\mathrm{p})=\left[\begin{array}{lll}
G_{O K}(\mathrm{p}) & G_{O I}(\mathrm{p}) & G_{O D}(\mathrm{p})
\end{array}\right]
$$

such that (1) can be written

$$
y_{O}=G_{O K}(\mathrm{p}) u_{K}+G_{O I}(\mathrm{p}) u_{I}+G_{O D}(\mathrm{p}) u_{D}+\underbrace{H_{O}(\mathrm{p}) \tau}_{\tau_{O}}
$$

which can be seen in Figure 1(c). Note that the model given by (2) and (5) is fairly general since both $u$ and $y_{O}$ are dependent on $\tau$, and the dependencies are controlled by the elements of $H_{O}(\mathrm{p})$ and $F_{\tau}(\mathrm{p})$. For example, in an open-loop experiment, the input is independent of the disturbance $\tau$ which implies that $F_{\tau}(\mathrm{p})=0$ (inputs are dependent on $\tau$ and $\delta$ through (2) and (3p). However, the framework also allows the system $G_{O}(\mathrm{p})$ to be part of a bigger more complicated system, such as a system operating in closed-loop (Forssell \& Ljung, 1999) or a module in a dynamic network (Van den Hof et al., 2013) where $F_{\tau}(\mathrm{p})$ is dependent on the controller and interconnection structure, respectively. This generality will be discussed further in Section 3.3 .

The partitioning of the transfer function matrix $G_{O}(\mathrm{p})$ is only one step on the way towards the indirect model. There are no assumptions made on this division, for instance, the individual transfer functions could share dynamics. This could be considered in the modeling process, for instance, by choosing joint parameters, or it could be neglected which would result in a model of higher order.

\section{The Indirect Framework - Elimination of Unknown Inputs}

One of the major issues with estimating a model of the subsystem $G_{O}(\mathrm{p})$ is the unknown inputs. It is assumed that some of these inputs $u_{D}$ can be measured through some known dynamics with an additive disturbance. These measurements are called direct input measurements and are given by

$$
y_{D}=G_{D}(\mathrm{p}) u+H_{D}(\mathrm{p}) \tau=G_{D D}(\mathrm{p}) u_{D}+\underbrace{H_{D}(\mathrm{p}) \tau}_{\tau_{D}}
$$

where $G_{D D}(\mathrm{p})$ is transfer function matrix of suitable dimension which is assumed to be known, $H_{D}(\mathrm{p})$ is a transfer function matrix and $y_{D}$ is a subset of the measurements $y$.

The remaining unknown inputs are only indirectly measured through the set of measurements

$$
y_{I}=G_{I}(\mathrm{p}) u+H_{I}(\mathrm{p}) \tau=G_{I K}(\mathrm{p}) u_{K}+G_{I I}(\mathrm{p}) u_{I}+G_{I D}(\mathrm{p}) u_{D}+\underbrace{H_{I}(\mathrm{p}) \tau}_{\tau_{I}}
$$

where the transfer functions matrices are of suitable dimensions and are possibly unknown and $y_{I}$ is a subset of $y$. The measurements $y_{I}$ are called indirect input measurements since 
they are dependent on the unknown inputs $u_{I}$ and thus indirectly contain information about these inputs.

Assumption 1: The transfer function matrices $G_{D D}(p)$ and $G_{I I}(p)$ are assumed to have full column rank. In addition, the transfer function matrix $G_{D D}(p)$ is assumed to be known. Note that the rank assumptions implies that, firstly, $\operatorname{dim}\left(y_{D}\right) \geq n_{D}$ and $\operatorname{dim}\left(y_{I}\right) \geq n_{I}$, respectively, and secondly, that the input measurements contain information about all the unknown inputs.

The categorization of the unknown inputs is based on the properties of the input measurements and there is a fundamental difference between the direct and indirect input measurements. To understand this difference, consider the case where the input measurements are noiseless. Then a known filter and the direct input measurement $y_{D}$ can be used directly to represent the unknown input $u_{D}$. In contrast, the indirect input measurements $y_{I}$ cannot be used without additional information, such as knowledge of the unknown dynamics and/or additional signals, to represent the unknown input $u_{I}$ even if the input measurements are noiseless.

\subsection{Eliminating Unknown Inputs Using Direct and Indirect Input Measurements}

The central idea of the indirect modeling framework is that the direct and indirect input measurements (6) and (7) can be used to eliminate the unknown inputs of (5) by algebraic manipulation. It is assumed that there exist two linear filters $f_{D D}(\mathrm{p})$ and $f_{I I}(\mathrm{p})$ of suitable dimensions, such that

$$
f_{D D}(\mathrm{p}) G_{D D}(\mathrm{p})=I_{n_{D} \times n_{D}}
$$

and

$$
f_{I I}(\mathrm{p}) G_{I I}(\mathrm{p})=I_{n_{I} \times n_{I}},
$$

where $I_{k \times k}$ is a $k$ by $k$ identity matrix.

The directly measured input $u_{D}$ is retained if (6) is solved for $G_{D D}(\mathrm{p}) u_{D}$ and filtered by $f_{D D}(\mathrm{p})$, i.e.

$$
u_{D}=f_{D D}(\mathrm{p})\left[y_{D}-H_{D}(\mathrm{p}) \tau\right]
$$

In the same way, $u_{I}$ is retained if $(7)$ is solved for $G_{I I}(\mathrm{p}) u_{I}$ and filtered by $f_{I I}(\mathrm{p})$ which results in

$$
u_{I}=f_{I I}(\mathrm{p})\left[y_{I}-G_{I K}(\mathrm{p}) u_{K}-G_{I D}(\mathrm{p}) u_{D}-H_{I}(\mathrm{p}) \tau\right]
$$

Finally, by inserting (10) and (11) into (5) we obtain the indirect model

$$
\begin{aligned}
y_{O} & =\tilde{G}_{O K}(\mathrm{p}) u_{K}+\tilde{G}_{O I}(\mathrm{p}) y_{I}+\tilde{G}_{O D}(\mathrm{p}) y_{D}+\tilde{H}_{O}(\mathrm{p}) \tau \\
& =\tilde{G}_{O}(\mathrm{p}) \tilde{u}+\bar{\tau}
\end{aligned}
$$

where 


$$
\begin{aligned}
& \tilde{u}=\left[\begin{array}{lll}
u_{K}^{T} & y_{I}^{T} & y_{D}^{T}
\end{array}\right]^{T}, \\
& \tilde{G}_{O}(\mathrm{p})=\left[\begin{array}{lll}
\tilde{G}_{O K}(\mathrm{p}) & \tilde{G}_{O I}(\mathrm{p}) & \tilde{G}_{O D}(\mathrm{p})
\end{array}\right], \\
& \tilde{G}_{O I}(\mathrm{p})=G_{O I}(\mathrm{p}) f_{I I}(\mathrm{p}) \text {, } \\
& \tilde{G}_{O K}(\mathrm{p})=G_{O K}(\mathrm{p})-\tilde{G}_{O I}(\mathrm{p}) G_{I K}(\mathrm{p}), \\
& \tilde{G}_{O D}(\mathrm{p})=\left(G_{O D}(\mathrm{p})-\tilde{G}_{O I}(\mathrm{p}) G_{I D}(\mathrm{p})\right) f_{D D}(\mathrm{p}),
\end{aligned}
$$

and

$$
\tilde{H}_{O}(\mathrm{p})=H_{O}(\mathrm{p})-\tilde{G}_{O I}(\mathrm{p}) H_{I}(\mathrm{p})-\tilde{G}_{O D}(\mathrm{p}) H_{D}(\mathrm{p})
$$

In Linder and Enqvist (2015), $G_{D D}(\mathrm{p})$ and $G_{I I}(\mathrm{p})$ were both assumed to be invertible. This implies that the dimension of the input measurements is equal to the number of unknown inputs. If there are additional input measurements, this invertibility assumption might be limiting since the extra measurements possibly can be used to improve the accuracy. Here, this assumption is relaxed and by Assumption 1, $G_{D D}(\mathrm{p})$ and $G_{I I}(\mathrm{p})$ are both assumed to have full column rank which is equivalent to the invertibility assumption if they are square matrices (Lancaster \& Tismenetsky, 1985). If $\operatorname{dim}\left(y_{D}\right)=n_{D}$ and $\operatorname{dim}\left(y_{I}\right)=n_{I}$, then the filters can be chosen as $f_{D D}(\mathrm{p})=G_{D D}^{-1}(\mathrm{p})$ and $f_{I I}(\mathrm{p})=G_{I I}^{-1}(\mathrm{p})$ which is equivalent to Linder and Enqvist (2015).

Furthermore, observe that in contrast to, for instance, blind identification Abed-Meraim et al., 1997), no assumptions have to be made on the unknown inputs since they have been eliminated from the indirect model through the algebraic manipulations. In fact, except for Assumption 1 there are no additional assumptions, such as stability, on the indirect model (12). We have intentionally chosen not to state such requirements since they could be limiting and lead to a conservative set of models. However, in practice, additional requirements might be necessary depending on the method chosen for estimation of the indirect model. With this in mind, it is important to realize that

(1) Given a certain system/model of the system, only some estimation methods might be suitable due to properties of the resulting indirect model.

(2) Given a specific estimation method, additional requirements might have to be imposed on the indirect model.

At this point, it might be worth noting that the proposed indirect model only has known inputs, but that the elimination of the unknown inputs results in new challenges due to the disturbances on the input measurements and these disturbances have to be considered while estimating the model.

If the input $\tilde{u}$ and the disturbance $\tau_{O}$ on the output are uncorrelated, the remaining disturbances $\tau_{D}$ and $\tau_{I}$ of the input measurements will lead to an errors-in-variables formulation. If the disturbances $\tau_{O}$ on the other hand are correlated with the inputs $y_{I}$ and $y_{D}$ of the indirect model, then regardless whether the original system is open loop, the indirect estimation problem shares the challenges of a closed-loop estimation problem.

The indirect model can in some sense be viewed as an artificial closed-loop system, with loops induced by using the measurements as inputs. Unlike a system with a real controller in the loop, the artificial loops of the indirect model might contain direct terms (Linder, Enqvist, \& Gustafsson, 2014). These direct terms are potentially a problem and some closed-loop identification methods, such as the direct prediction error method, might fail if this is not considered (Dankers, 2014). 


\subsection{Predictor Input and Output Selection}

In Sections 2 and 3 , the division of the measurements $y$ into the output $y_{O}$ and the input measurements $y_{D}$ and $y_{I}$ were assumed to be given. In practice, this division has to be done by the user. Given the desirable dynamics or properties, the measurements $y$ and the external signals $\delta$ and $\tau$, it is not always obvious which subsystem that should be estimated, i.e. how to divide the measurements. This ambiguity stems partially from the symmetry of (5) and (7) which is illustrated by the following example.

Example 1 (Ambiguity in predictor in- and output selection): The choice of output $y_{O}$ and input measurement $y_{I}$ is not always simple and is dependent on the application. Consider the model

$$
y=\left[\begin{array}{l}
y_{1} \\
y_{2}
\end{array}\right]=\left[\begin{array}{l}
G_{1}(\mathrm{p}) \\
G_{2}(\mathrm{p})
\end{array}\right]\left[\begin{array}{l}
u_{K} \\
u_{I}
\end{array}\right]=\left[\begin{array}{l}
G_{1 K}(\mathrm{p}) \\
G_{2 K}(\mathrm{p})
\end{array}\right] u_{K}+\left[\begin{array}{l}
G_{1 I}(\mathrm{p}) \\
G_{2 I}(\mathrm{p})
\end{array}\right] u_{I}
$$

where $y_{1}, y_{2}, u_{K}$ and $u_{I}$ are all scalar. We are interested in the dynamics of $G_{1}(\mathrm{p})$ and both measurements can be used as input measurement. If the second measurement is used as input measurement, the indirect model is given by

$$
y_{1}=\left(G_{1 K}(\mathrm{p})-G_{1 I}(\mathrm{p}) G_{2 I}^{-1}(\mathrm{p}) G_{2 K}(\mathrm{p})\right) u_{K}+G_{1 I}(\mathrm{p}) G_{2 I}^{-1}(\mathrm{p}) y_{2}
$$

Note that if 20$)$ is multiplied by $\left(G_{1 I}(\mathrm{p}) G_{2 I}^{-1}(\mathrm{p})\right)^{-1}=G_{2 I}(\mathrm{p}) G_{1 I}^{-1}(\mathrm{p})$, then

$$
y_{2}=\left(G_{2 K}(\mathrm{p})-G_{2 I}(\mathrm{p}) G_{1 I}^{-1}(\mathrm{p}) G_{1 K}(\mathrm{p})\right) u_{K}+G_{2 I}(\mathrm{p}) G_{1 I}^{-1}(\mathrm{p}) y_{1}
$$

is obtained after some algebraic manipulations. Equation (21) is the model given if the first measurement is used as input measurement. Estimating the model $(20)$ is thus in some sense the inverse problem to estimating the one in (21). Which formulation that is preferable depends on the application, for instance, in estimation of inverse models it might be beneficial to directly estimate the inverse rather than to first estimate a model and then inverting it, see Jung and Enqvist (2013).

The choice of predictor inputs and outputs can be based on properties of the resulting indirect model, such as stability of the predictor or that the transfer function matrices are proper. Or the choice might depend on the usage of the model as was briefly mentioned in Example 1. Hence, the division of measurements into output $y_{O}$ and the input measurements $y_{D}$ and $y_{I}$ depends on the application and ultimately, many choices can lead to satisfactory results.

\subsection{Connection to Existing System Identification Problems}

One strength of the indirect model framework is the flexibility. In addition to solving a new set of system identification problems, many special cases of the indirect model (12), such as when there are only indirectly measured inputs, are equivalent to already existing system identification problems and (12) can in this sense be seen as a generalization of these classical problems.

The most elementary case is when $F_{\tau}=0$ and hence, that the input $u$ defined in $(2)$ is uncorrelated with the disturbance $\tau$, i.e. that (5) is in open-loop operation. Let us look at 


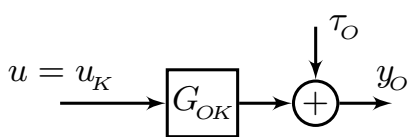

(a) Open-loop estimation.

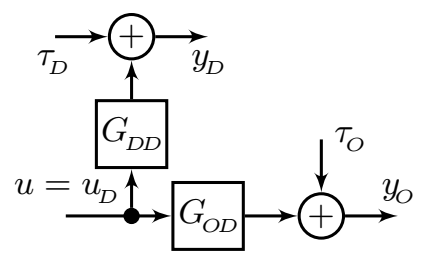

(b) Open-loop EIV estimation.

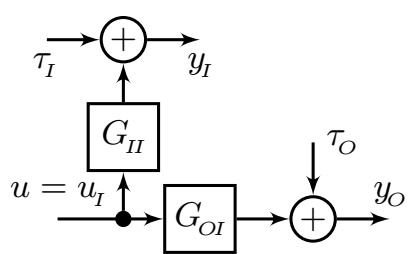

(c) Open-loop S2SID estimation.

Figure 2. In some sense, the indirect framework is a generalization of existing system identification problems. The classical open-loop problem (a), the classical EIV problem (b) and the S2SID problem (c) are all special cases if the system is acting in open loop $\left(F_{\tau}=0\right)$.

three existing problems:

(a) If the dimension of $u$ is equal to the number of known inputs $n_{K}$, then the classical open-loop case shown in Figure 2(a) is recovered. See, for instance, Ljung (1999) for the discrete-time case and Garnier and Wang (2008) for the related continuous-time counterpart.

(b) If all the inputs are directly measured, i.e. $n_{D}=\operatorname{dim}(u)$, then the indirect model will result in an errors-in-variable (EIV) problem, see Figure 2(b), The classical EIV problem is given if $G_{D D}=1$ and $\tau_{D}$ is measurement noise. Note that the framework of this paper allows $\tau_{O}$ and $\tau_{D}$ to be correlated and that extra consideration have to be taken in this case. Söderström (2007) gives a survey for EIV problems and Mahata and Garnier (2006) deals with one method of estimating the model in a continuous-time EIV problem.

(c) Finally, if $n_{I}=\operatorname{dim}(u)$ and hence, that there are only indirectly measured inputs, then the resulting model is a sensor-to-sensor system identification problem (S2SID) and the resulting transfer function is called a pseudo transfer function (D'Amato et al., 2009). Note that the principal structure of the S2SID problem shown in Figure 2(c) is misleadingly similar to the EIV problem shown in Figure 2(b). However, there is a significant difference not shown in the superficial structures of the problems in Figures 2(b) and $2(\mathrm{c})$. In the S2SID framework, dynamics shared between $G_{I I}(\mathrm{q})$ and $G_{O I}(\mathrm{q})$ will cancel. To emphasize the difference, let us consider an example.

Example 2 (Difference between EIV and S2SID): Consider the models

$$
y_{1}=G_{1}(\mathrm{p}) u=\bar{G}_{1}(\mathrm{p}) G(\mathrm{p}) u \quad \text { and } \quad y_{2}=G_{2}(\mathrm{p}) u=\bar{G}_{2}(\mathrm{p}) G(\mathrm{p}) u
$$

i.e. that the transfer functions share dynamics. If both the transfer functions and the input $u$ are unknown, the problem can be put into the S2SID framework. The shared dynamics $G(\mathrm{p})$ can never be estimated in the S2SID framework since the pseudo transfer functions are

$$
y_{1}=\bar{G}_{1}(\mathrm{p}) \bar{G}_{2}^{-1}(\mathrm{p}) y_{2} \quad \text { or } \quad y_{2}=\bar{G}_{2}(\mathrm{p}) \bar{G}_{1}^{-1}(\mathrm{p}) y_{1}
$$

However, if either $G_{1}(\mathrm{p})$ or $G_{2}(\mathrm{p})$ would be known, then the problem can instead be considered an EIV problem with $G_{D D}(\mathrm{p})$ equal to the known transfer function. This means that $G(\mathrm{p})$ can be estimated as part of $G_{O D}(\mathrm{p})$.

More flexible models are obtained for the case when $F_{\tau} \neq 0$, which means that the input $u$ defined in (2) is correlated with the disturbance $\tau$, i.e. that there is some feedback mechanism 


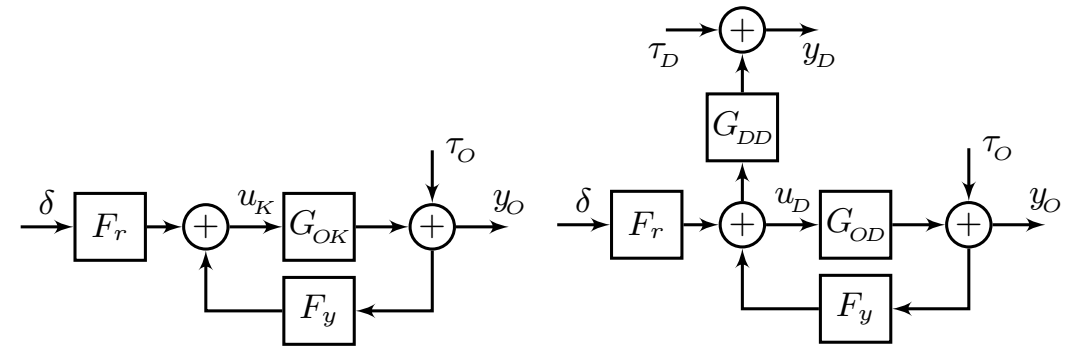

(a) Closed-loop estimation.

(b) Closed-loop EIV estimation.

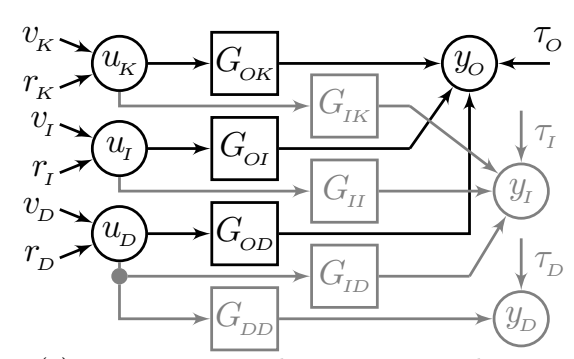

(c) Estimation in dynamic networks.

Figure 3. Allowing $F_{\tau}$ to be nonzero allows increasingly complex systems to be modeled.

from the measurements $y$ to the input $u$. Three examples of special cases are:

(d) Figure 3(a) shows the classical closed-loop system identification problem described by

$$
y_{O}=G_{O K}(\mathrm{p}) u_{K}+H_{O}(\mathrm{p}) \tau \text { and } u_{K}=F_{y}(\mathrm{p}) y_{O}+F_{r}(\mathrm{p}) \delta
$$

where the input $u$ is assumed to be known, i.e. $n_{K}=\operatorname{dim}(u)$. If the equations of (24) are combined, the input is given by

$$
u_{K}=\underbrace{\left(I-F_{y}(\mathrm{p}) G_{O K}(\mathrm{p})\right)^{-1} F_{y}(\mathrm{p}) H_{O}(\mathrm{p})}_{F_{\tau}(\mathrm{p})} \tau+\underbrace{\left(I-F_{y}(\mathrm{p}) G_{O K}(\mathrm{p})\right)^{-1} F_{r}(\mathrm{p})}_{F_{\delta}(\mathrm{p})} \delta
$$

which fits the indirect framework. For details on closed-loop system identification, see for instance, Forssell and Ljung (1999).

(e) Similarly, an EIV closed-loop system identification problem is recovered if the input is directly measured $\left(u=u_{D}\right)$. For more details on the EIV closed-loop problem, see for instance, Söderström et al. (2013).

(f) An even more general type of model is a dynamic network where the closed-loop problems (d) and (e) are special cases. In the dynamic network framework of Van den Hof et al. (2013) and Dankers (2014), there are a number of internal signals (nodes) and a subset of these nodes are assumed to be measured. The nodes are dynamically dependent on each other and there are external user-controllable signals and unmeasured disturbances possibly entering at each node. A dynamic network model can be cast into the indirect framework and the equations (2), (5), (6) and (7) can be written as the network model

$$
\left[\begin{array}{l}
y_{O} \\
y_{I} \\
y_{D} \\
\hdashline u_{K} \\
\hdashline u_{I} \\
u_{D}
\end{array}\right]=\left[\begin{array}{ccc:c:cc}
0 & 0 & 0 & G_{O K}(\mathrm{p}) & G_{O I}(\mathrm{p}) & G_{O D}(\mathrm{p}) \\
0 & 0 & 0 & G_{I K}(\mathrm{p}) & G_{I I}(\mathrm{p}) & G_{I D}(\mathrm{p}) \\
0 & 0 & 0 & 0 & 0 & G_{D D}(\mathrm{p}) \\
\hdashline 0 & 0 & 0 & 0 & 0 & 0 \\
\hdashline 0 & 0 & 0 & 0 & 0 & 0 \\
0 & 0 & 0 & 0 & 0 & 0
\end{array}\right]\left[\begin{array}{c}
y_{O} \\
y_{I} \\
y_{D} \\
\hdashline u_{K} \\
\hdashline u_{I} \\
u_{D}
\end{array}\right]+\left[\begin{array}{c}
0 \\
0 \\
0 \\
\hdashline F_{\delta}(\mathrm{p})
\end{array}\right] \delta+\left[\begin{array}{c}
H_{O}(\mathrm{p}) \\
H_{I}(\mathrm{p}) \\
H_{D}(\mathrm{p}) \\
F_{\tau}(\mathrm{p}) \\
\end{array}\right] \tau
$$

which can also be seen in Figure $3(\mathrm{c})$ (where the notation $r=F_{\delta}(\mathrm{p}) \delta$ and $v=F_{\tau}(\mathrm{p}) \tau$ has been introduced) where the desired dynamics is indicated with black while the input measurements are drawn in grey. Note that the input measurements $y_{D}$ and $y_{I}$ cannot be used directly in the framework of Dankers (2014) to estimate the transfer function $G_{O}(\mathrm{p})$ since there is no path from the input measurements $y_{I}$ and $y_{D}$ to the output $y_{O}$. In some sense, the use of the input measurements and the transformation into the indirect model (12) create artificial paths that make it possible to utilize these measurements. The input measurements $y_{D}$ and $y_{I}$ can with this point of view be seen as an generalization of the additive measurement error in the framework of Dankers (2014). 


\section{Possible Benefits of Using the Indirect Framework}

In this section, the necessity or benefit of including an input will be discussed in the context of identifiability, consistency and variance properties. Due to the complexity of the indirect model (and the fact that consistency and variance properties are dependent on the estimation method), it is difficult to draw conclusions in the general case. Instead, the discussion will be centered around simple and illustrative examples that highlight important aspects.

In order to facilitate the discussion, the models in this section are given a specific parameterization. The set of parameters denoted by $\vartheta$ is assumed to describe the desired dynamics or properties of the model. Regardless, if the indirect model $(12)$ is parameterized directly or if the desired model (5) and the input measurement models (6) and (7) are first parameterized to then form the indirect model (12), it is assumed that they are all dependent on the parameters $\vartheta$.

\subsection{Identifiability Properties of the Indirect Model}

Once a parameterization of the model has been chosen, an initial step should be to investigate the identifiability properties of the indirect model. In this section, the focus is the structural identifiability (henceforth called identifiability), i.e. if it is possible to uniquely determine the parameters in the chosen model structure (Bazanella, Gevers, \& Miškovic, 2010; Bellman \& Åström, 1970; Ljung \& Glad, 1994). Note that the models defined in Sections 2 and 3 are assumed to be linear in the inputs but that there is no linearity assumption on the parameterization. For details on identifiability analysis of linear systems with nonlinear parameterizations, see for instance, Hermann and Krener (1977), Walter (1982), Diop and Fliess (1991) and Anguelova (2007). It is difficult to give an explicit condition for the identifiability of the resulting indirect model $(12)$ given an arbitrary nonlinear parameterization of (5). However, there are some aspects worth considering.

Firstly, consider the most straightforward case when $G_{O K}(\mathrm{p}, \vartheta)$ only depends on a subset of the parameters. The predictor where the inputs $u_{D}$ and $u_{I}$ are neglected, would thus not be dependent on all parameters and would then trivially not be identifiable.

The use of input measurement can thus avoid trivial identifiability issues. But the input measurements can also contribute with new information and it is not necessary that the original model (5) is identifiable with respect to the parameters. To explore this, let us look at an example.

Example 3 (Gaining identifiability from input measurement): Consider the simple static model

$$
y_{O}=b_{1} u_{K}+b_{2}^{2} u_{I}
$$

where the goal is to estimate both $b_{1}$ and $b_{2}$. Since $b_{2}$ only appears squared, it is impossible to distinguish between $b_{2}=b_{2}^{0}$ and $b_{2}=-b_{2}^{0}$ where $b_{2}^{0}$ is the true value of the parameter. This implies that the model is not identifiable even if both inputs are known.

However, if the indirect input measurement $y_{I}=b_{2} u_{I}$ is available, then the resulting indirect model

$$
y_{O}=b_{1} u_{K}+b_{2} y_{I}
$$

is identifiable with respect to the parameters. 
Unfortunately, identifiability might also be lost if the input measurement shares dynamics or parameters with the desired system as discussed in Example 2. Let us again look at an example.

Example 4 (Loss of identifiability due to shared dynamics): Consider the simple static model

$$
y_{O}=b_{1} u_{K}+b_{2} u_{I}
$$

where the goal is to estimate the parameters $b_{1}$ and $b_{2}$. Both parameters would be identifiable if the inputs were known. However, as in Example 3, the input $u_{I}$ is unknown but the indirect input measurement $y_{I}=b_{2} u_{I}$ is available. The resulting indirect model

$$
y_{O}=b_{1} u_{K}+y_{I}
$$

is not identifiable with respect to $b_{2}$ due to cancelations when the indirect model is formed.

However, note that even if the original model (29) is identifiable, it does not imply that we can practically estimate both parameters since $u_{I}$ is unknown. Hence, the loss of identifiability is in relation to the original model (29) where all inputs are known.

In conclusion, it is the indirect model (12) that has to be identifiable with respect to the parameters and neglecting inputs might lead to trivial losses of identifiability as discussed above. If (5), (6) and (7) are first parameterized, identifiability of (5) might both be gained (or lost) when the indirect model (12) is formed due to shared dynamics or parameters with the input measurements.

\subsection{Consistency and Variance Properties of the Indirect Model}

As stated in Section 2, it is assumed that all contributions from the external user-controllable signal $\delta$ to $y_{O}$ are captured in the input $u$. This implies that there are no paths from the external signal $\delta$ to the output $y_{o}$ not passing through the input $u$. The necessity of including an input depends on the correlation between the inputs. In this section, the simple system with one known and one unknown input shown in Figure 4 will be used in three examples to illustrate some important aspects. In each example, the goal is to estimate $b_{K}$ and we will look at the consistency and variance properties of the estimator. The first example will establish a baseline to see what is possible if all inputs are known. In the second example, the unknown input will be neglected and in the third example an indirect input measurement will be used.

Here, the covariance of the parameter estimate $\hat{\vartheta}$ is given by $\operatorname{Cov}(\hat{\vartheta})=P_{\vartheta} / N$ where $P_{\vartheta}$ is the covariance of the asymptotic distribution. Furthermore, $\vartheta^{*}$ will be used to represent the limit value, i.e. $\hat{\vartheta} \rightarrow \vartheta^{*}$ with probability 1 as the number of data points tends towards infinity, see Ljung (1999, Ch. 9) for more details.

Example 5 (Static system with unknown input): To get some insight into the problem consider the static system shown in Figure 4 where the goal is to estimate the parameter $b_{K}$ accurately. The system is described by

$$
y_{O}=b_{K} u_{K}+b_{I} u_{I}+\tau_{O}
$$


where $b_{K}$ and $b_{I}$ are constant parameters and $\tau_{O} \sim \mathcal{N}\left(0, \lambda_{O}\right)$. The input is given by

$$
\left[\begin{array}{l}
u_{K} \\
u_{I}
\end{array}\right]=\left[\begin{array}{ll}
1 & 0 \\
\alpha & 1
\end{array}\right]\left[\begin{array}{l}
\delta_{1} \\
\delta_{2}
\end{array}\right]
$$

where $\alpha$ is a constant, $\delta_{1} \sim \mathcal{N}\left(0, \lambda_{1}\right)$ and $\delta_{2} \sim \mathcal{N}\left(0, \lambda_{2}\right)$. Here, $\mathcal{N}(0, \lambda)$ describes Gaussian noise with zero mean and variance $\lambda$. Assuming that both inputs are known, consider the predictor

$$
\hat{y}_{O}=\hat{b}_{K} u_{K}+\hat{b}_{I} u_{I}
$$

where the parameters are found using a least-squares criterion. This results in the estimates $b_{K}^{*}=b_{K}$ and $b_{I}^{*}=b_{I}$ with the asymptotic covariance given by

$$
P \underset{\vartheta}{5}=\left[\begin{array}{cc}
P \sqrt[5]{5} & \times \\
\times & \times
\end{array}\right]=\lambda_{O}\left[\begin{array}{cc}
\frac{\alpha^{2}}{\lambda_{2}}+\frac{1}{\lambda_{1}} & -\frac{\alpha}{\lambda_{2}} \\
-\frac{\alpha}{\lambda_{2}} & \frac{1}{\lambda_{2}}
\end{array}\right]
$$

which implies that increasing the power of $\delta_{2}$ will improve the accuracy also of $\hat{b}_{K}$ unless $\alpha=0$.

Example 5 shows classical results for the least-squares estimator, see for instance, Ljung (1999). To show the importance of including all inputs, let us first look at consistency and variance properties in an example where one input is neglected in the estimator.

Example 6 (Consequences of neglecting input): Let us again consider the problem of Example 5 but now assume that $u_{I}$ is unknown and look at what happens if it is neglected. Since the goal is to estimate $b_{K}$ with good accuracy, let us instead try the simple predictor model

$$
\hat{y}_{O}(\vartheta)=\hat{b}_{K} u_{K}
$$

which is identifiable with respect to the desired parameter and where the parameter again is found by minimizing a least-squares criterion. The resulting estimate is given by $b_{K}^{*}=b_{K}+\alpha b_{I}$, which is biased when $\alpha \neq 0$.

The asymptotic variance is given by

$$
P_{b_{K}}^{6}=\frac{b_{I}^{2} \lambda_{2}+\lambda_{O}}{\lambda_{1}}
$$

which is independent of the correlation between the inputs. Note that in contrast to Exam-

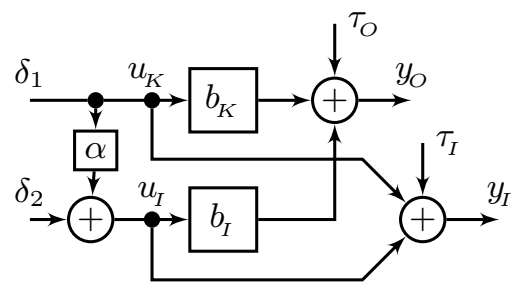

Figure 4. A static system is dependent on one known and one unknown input. The inputs are dependent on the external signals $\delta_{1}$ and $\delta_{2}$ and the output is measured with noise. In conjunction to the output measurement, there is also a noisy indirect input measurement. 
ple 5 , increasing $\lambda_{2}$ will decrease the accuracy of $\hat{b}_{K}$ regardless of $\alpha$. This is not surprising since the predictor describes less of the output.

Note that, the least-squares predictor used in Example 6 is not the cause of the bias. The reason is that there is a parallel path from $\delta_{1}$ to $y_{O}$ that is not included in $\hat{y}_{O}$, i.e. it is the correlation of $u_{K}$ and $u_{I}$ in Example 6 that introduces the bias. Hence, it is necessary to include the unknown input to avoid bias in the correlated case. Let us now see what can be achieved with the indirect model.

Example 7 (Using indirect input measurement): Let us once again consider the problem in Example 5 where the goal is to estimate $b_{K}$ but now $u_{I}$ is assumed to be unknown. However, there is an input measurement given by

$$
y_{I}=u_{K}+u_{I}+\tau_{I}
$$

where $\tau_{I} \sim \mathcal{N}\left(0, \lambda_{I}\right)$ is measurement noise. Solving (37) for $u_{I}$ and inserting into (31) results in the indirect predictor model given by

$$
\hat{y}_{O}=\left(\hat{b}_{K}-\hat{b}_{I}\right) u_{K}+\hat{b}_{I} y_{I}
$$

which is identifiable with respect to the desired parameter $b_{K}$. Using the predictor (38) together with a least-squares criterion results in the estimate

$$
\vartheta^{*}=\left[\begin{array}{c}
b_{K} \\
0
\end{array}\right]+\left[\begin{array}{c}
\frac{\alpha \lambda_{I}}{\lambda_{2}+\lambda_{I}} b_{I} \\
\frac{\lambda_{2}}{\lambda_{2}+\lambda_{I}} b_{I}
\end{array}\right]
$$

Note that a bias is introduced due to using a noisy input in the least-squares estimator. The bias is dependent on the signal to noise ratio of the indirect input measurement, i.e. it will decrease if $\lambda_{2}$ is increased or $\lambda_{I}$ is decreased. Furthermore, the bias $\alpha b_{I}$ if the input is neglected will always be larger than or equal to the bias $\alpha b_{I} \lambda_{I} /\left(\lambda_{2}+\lambda_{I}\right)$ of the indirect estimator since $\lambda_{I} /\left(\lambda_{2}+\lambda_{I}\right) \leq 1$.

The asymptotic covariance is given by

$$
P P_{\vartheta}^{7}=\left[\begin{array}{cc}
P_{b_{K}}^{7} & \times \\
\times & \times
\end{array}\right]=\left[\begin{array}{cc}
\frac{b_{I}{ }^{2} \lambda_{2}}{\lambda_{1}} \cdot \frac{\lambda_{I}\left(\alpha^{2} \lambda_{1}+\lambda_{2}+\lambda_{I}\right)}{\left(\lambda_{2}+\lambda_{I}\right)^{2}}+\lambda_{O}\left(\frac{\alpha^{2}}{\lambda_{2}+\lambda_{I}}+\frac{1}{\lambda_{1}}\right) & -\frac{\alpha\left(\lambda_{2} \lambda_{I} b_{I}{ }^{2}+\lambda_{2} \lambda_{O}+\lambda_{I} \lambda_{O}\right)}{\left(\lambda_{2}+\lambda_{I}\right)^{2}} \\
-\frac{\alpha\left(\lambda_{2} \lambda_{I} b_{I}{ }^{2}+\lambda_{2} \lambda_{O}+\lambda_{I} \lambda_{O}\right)}{\left(\lambda_{2}+\lambda_{I}\right)^{2}} & \frac{\lambda_{2} \lambda_{I} b_{I}{ }^{2}+\lambda_{2} \lambda_{O}+\lambda_{I} \lambda_{O}}{\left(\lambda_{2}+\lambda_{I}\right)^{2}}
\end{array}\right]
$$

In a variance sense, there is a benefit of using the indirect model if the variance $P \frac{7}{b_{K}}$ is smaller than the variance $P_{b_{K}}^{6}$ (of Example 6 ) when the input is neglected, i.e.

$$
\frac{b_{I}^{2} \lambda_{2}}{\lambda_{1}} \cdot \frac{\lambda_{I}\left(\alpha^{2} \lambda_{1}+\lambda_{2}+\lambda_{I}\right)}{\left(\lambda_{2}+\lambda_{I}\right)^{2}}+\lambda_{O}\left(\frac{\alpha^{2}}{\lambda_{2}+\lambda_{I}}+\frac{1}{\lambda_{1}}\right)<\frac{b_{I}^{2} \lambda_{2}+\lambda_{O}}{\lambda_{1}}
$$

which is equivalent to

$$
\underbrace{\left(\alpha^{2} \lambda_{O} \lambda_{1}\right.}_{C_{1}}) \lambda_{2}-(\underbrace{\left.b_{I}^{2}\right)}_{C_{2}} \lambda_{2}^{3}+\lambda_{I}[\underbrace{\left(\alpha^{2} \lambda_{O} \lambda_{1}\right)}_{C_{3}}+\underbrace{\alpha^{2} b_{I}^{2} \lambda_{1}}_{C_{4}}) \lambda_{2}-\underbrace{\left(b_{I}^{2}\right)}_{C_{5}} \lambda_{2}^{2}]<0
$$

if $\lambda_{1}\left(\lambda_{2}+\lambda_{I}\right)^{2}>0$. 
If the parameter $b_{I}=0$ then it is always better to neglect the unknown input since it does not affect the output. On the other hand, if $b_{I} \neq 0$ and the inputs are independent, i.e. $\alpha=0$, then it is always useful to use the indirect input measurement since (42) is fulfilled and $b_{K}^{*}=b_{K}$. Hence, if $\lambda_{I}>0$ the indirect model will always give an unbiased estimator of $b_{K}$ with variance less than or equal to the case where $u_{I}$ was neglected.

In the general case where all parameters are non-zero, then all the coefficients $C_{i}, i=$ $1, \ldots, 5$, of (42) are non-negative. It is thus sufficient to consider the special case $\alpha=\lambda_{1}=$ $\lambda_{O}=b_{I}=1$ that captures the essence of the inequality. With these values, the inequality becomes

$$
\lambda_{2}-\lambda_{2}^{3}+\lambda_{I}\left(1+\lambda_{2}-\lambda_{2}^{2}\right)<0
$$

Note that if $1+\lambda_{2}-\lambda_{2}^{2}$ is negative, which is true for $\lambda_{2}>1 / 2+\sqrt{5 / 4} \approx 1.618$, then 43 is fulfilled for all values of $\lambda_{I}$. Figure $5(\mathrm{a})$ shows the contour of $P_{b_{K}}^{7 \sqrt[7]{5}}-P_{b_{K}}^{6}$, i.e. the differences in asymptotic variance of $\hat{b}_{K}$ between the estimators as a function of $\lambda_{2}$ and $\lambda_{I}$. The curve corresponding to $\lambda_{2}-\lambda_{2}^{3}+\lambda_{I}\left(1+\lambda_{2}-\lambda_{2}^{2}\right)=0$ (black line) and the line $\lambda_{2}=1.618$ (dashed black line) are also drawn. Here, (43) is fulfilled for all values to the right of the black line. For instance, it can be seen that if $\lambda_{I}=0$, then we need that $\lambda_{2}>1$ for the indirect model to give a better result than neglecting the unknown input. Furthermore, if $\lambda_{2}$ is small, it is better to neglect the unknown input.

Comparing variance properties of the two estimators can be seen as unfair since the estimators are biased. Figure 5(b) shows the asymptotic mean squared error (AMSE) of $\hat{b}_{K}$, i.e. taking both variance and bias into account, given by

$$
\operatorname{AMSE}\left(\hat{b}_{K}\right)=P_{b_{K}}+\left(b_{K}^{*}-b_{K}\right)^{2}
$$

In this case, the AMSE is smaller for the indirect model if $\lambda_{2}>0.62$ regardless of $\lambda_{I}$.

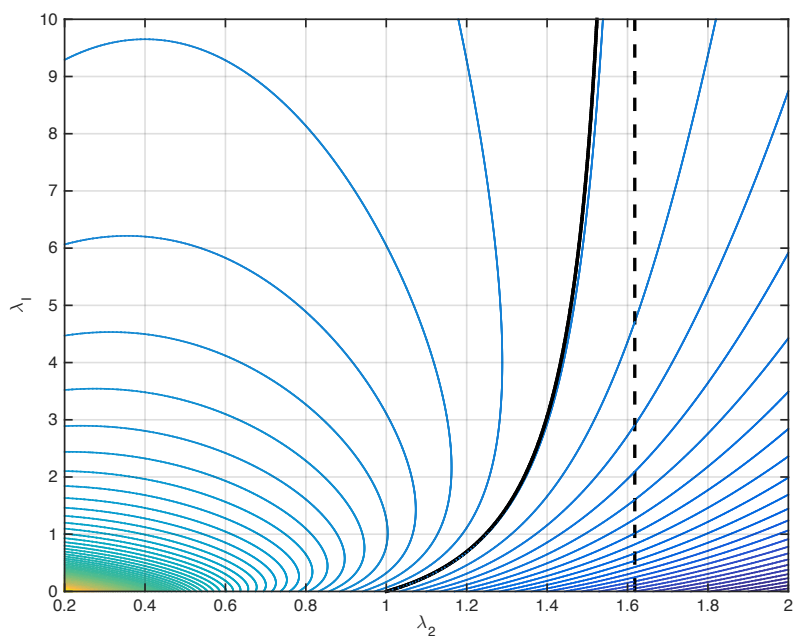

(a) The difference in variance of $\hat{b}_{K}$.

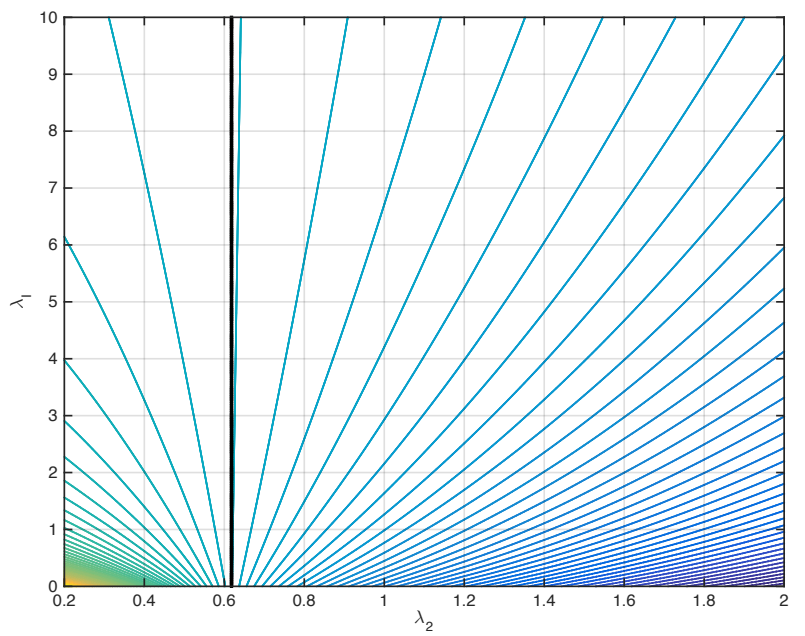

(b) The difference in AMSE of $\hat{b}_{K}$.

Figure 5. The differences between neglecting $u_{I}$ (Example 6) and using an indirect input measurement (Example 7 as a function of the input variance $\lambda_{2}$ and measurement noise of the indirect input measurement $\lambda_{I}$. The figures show the asymptotic variance/AMSE of $\hat{b}_{K}$ in Example 7 minus the asymptotic variance/AMSE of $\hat{b}_{K}$ in Example 6 The values to the right of the black line corresponds to when it is beneficial to use the indirect model. For values $\lambda_{2}>1 / 2+\sqrt{5 / 4} \approx 1.618$ (dashed line) it is always beneficial to use the indirect predictor regardless of the value of $\lambda_{I}$ in a variance sense. For instance, it can be seen that if the input measurement is noisefree, then the indirect model will perform better in a variance sense if $\lambda_{2}>1$. 
Note that, in contrast to Example 6, the bias of Example 7 is a result of the use of the leastsquares estimator. The bias of (39) decreases with decreasing measurement noise variance $\lambda_{I}$ of the input measurement and the estimator is consistent if $\lambda_{I}=0$. The influence of the noise can be mitigated with a proper choice of estimator. For example, the instrumental variable estimator that will be briefly described in Section 5 is consistent also for $\lambda_{I} \neq 0$.

In conclusion, an input can be neglected without affecting the consistency properties of the remaining input paths if it is independent of these inputs. Furthermore, by using the input measurements, consistency can be achieved with the correct choice of estimator. The variance properties of the indirect model are dependent on the signal to noise ratio of the input measurements as well as the unknown true system. It is difficult to make a general statement, but intuitively one can decrease the variance if the unknown input has a significant contribution to the output and the input measurements are sufficiently accurate.

\section{Experimental Verification}

In this section, an experimental study will be presented. The goal of this section is not to do an exhaustive study of the experiment, but rather to show how the indirect framework can be applied to a real-world problem and present one viable option to estimate the desired properties or dynamics.

The experiment is the modified inverted pendulum seen in Figure 6(b) and the goal is to estimate the change in mass and change in center of mass (CM) of the pendulum using inertial measurements. The pendulum example has simple and intuitive dynamics that is a good approximation of the roll dynamics of many vehicles, such as cars, trucks or ships. In addition to the pendulum setup, the framework has been successfully applied to estimate a scale-model ship's dynamics which is considerably more complex, see Linder, Enqvist, Fossen, Johansen, and Gustafsson (2015a) and (2015b).

In any modeling framework, there are a number of choices, such as continuous- or discretetime model or parameterization, that the user has to consider during modeling and the choices will straightforwardly decide the properties of the resulting model. Depending on these properties, there might be several methods to estimate the desired dynamics or properties. Here, we will focus on a discrete-time model parameterized with physical parameters and estimated using an instrumental variable (IV) method. Note that the indirect modeling framework is not dependent on the approach suggested or the choices made in this section. The approach and choices are merely one example and depending on the application, other alternatives might be more suitable as discussed in Section 3.1.

\subsection{The Ideal Modified Inverted Pendulum Experiment}

Figure 6(b) shows a modified inverted pendulum hinged on a cart. The goal is to estimate or track the mass and the center of mass (CM) over time. The mass of the pendulum is $\bar{M}$ and the $\mathrm{CM}$ is located a distance $\bar{z}_{g}$ from the center of rotation $(\mathrm{CR})$ and with the inertia $I_{x}$ around its CG. The pendulum is stabilized using two springs, see Figure 6(b). These springs, the pendulum joint and the cables attached to pendulum are assumed to introduce two torsional torques acting on the pendulum. The first restoring torque is linearly dependent on the angle $\phi$ and the second torque is linearly dependent on the angular velocity $\dot{\phi}$ and corresponds to friction. There is an external unknown force acting on the cart which results in the acceleration $a_{y}$. Finally, a torque disturbance $\tau$ is assumed to act directly on the 
pendulum. Here, a linear model is sufficient since we are expecting small angles and the model is given by

$$
\bar{I}_{1} \ddot{\phi}=\overbrace{-\left(k+\bar{M} g \bar{z}_{g}\right) \phi}^{\text {restoring torque }} \overbrace{-d \dot{\phi}}^{\text {friction }} \overbrace{+\bar{M} \bar{z}_{g} a_{y}}^{\text {unknown input }} \overbrace{+\tau}^{\text {disturbances }}
$$

where $\bar{I}_{1}=I_{x}+\bar{M} \bar{z}_{g}^{2}$. Note that it is a minor approximation to use a linear model since the angle of the pendulum was small during the experiment. Furthermore, there is an inertial measurement unit (IMU) mounted on the cart a distance $z_{s}$ from the CR and it is assumed to measure

$$
\begin{aligned}
& y_{1}=\dot{\phi}+b_{1}+e_{1} \\
& y_{2}=a_{s}+b_{2}+e_{2}=z_{s} \ddot{\phi}+g \phi-a_{y}+b_{2}+e_{2}
\end{aligned}
$$

where $a_{s}$ is the tangential acceleration, $b_{i}, i=1,2$, are sensor biases and $e_{i}, i=1,2$, are measurement noises. The IMU only measures the resulting motion of the unknown force pulling the cart. Without additional information, it is impossible to know if it is a heavy pendulum affected by a large force or a light pendulum affected by a small force. Here, we will overcome this challenge by introducing

$$
\bar{M}=M+m \quad \text { and } \quad \bar{z}_{g}=\left(M z_{g}+m z_{m}\right) / \bar{M}
$$

where $M$ is a known nominal mass, $z_{g}$ is a known nominal CM, $m$ is an unknown additional mass and $z_{m}$ is an unknown CM for the additional mass, together with a nominal dataset $Z^{N}$, see Linder, Enqvist, Gustafsson, and Sjöberg (2014). Inserting (47) into (45) results in the model

$$
I_{1} \ddot{\phi}=-\left(k+M g z_{g}+m g z_{m}\right) \phi-d \dot{\phi}+\left(M z_{g}+m z_{m}\right) a_{y}+\tau
$$

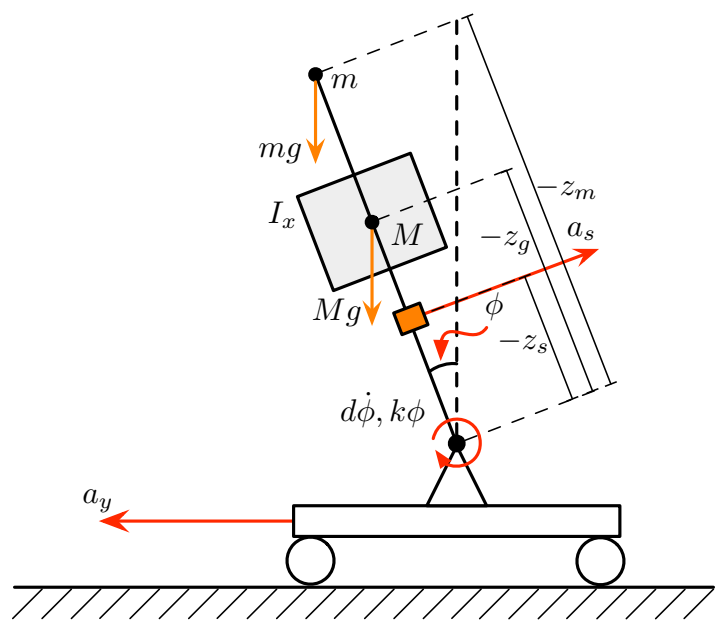

(a) An ideal sketch of the pendulum model showing the relation of the important quantities.

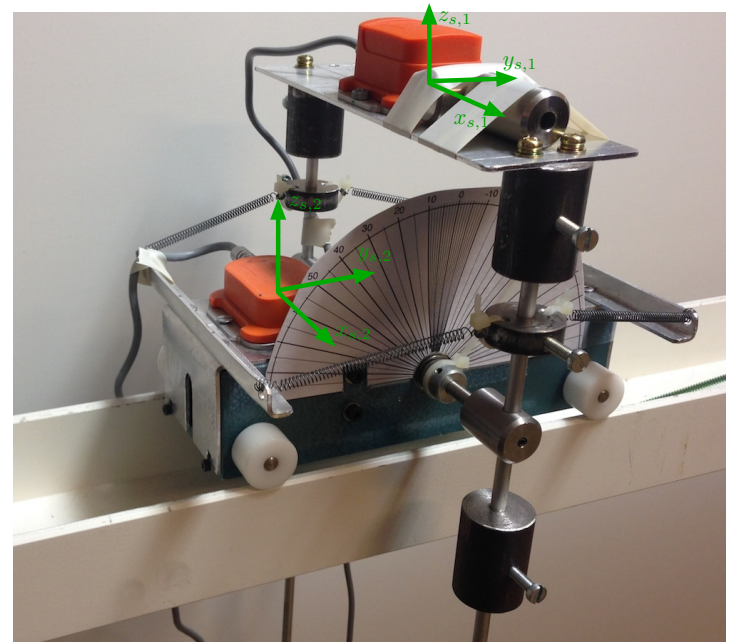

(b) Uppermost in the picture is the IMU (orange) measuring the rolling motion. In front of it is the additional load fastened with white tape. To the left on the cart is the reference IMU.

Figure 6. The modified inverted pendulum experimental setup. Note that the pendulum is stabilized with springs. 
where $I_{1}=I_{x}+M z_{g}^{2}+m z_{m}^{2}$ and an ideal sketch of the pendulum relating all the quantities can be seen in Figure 6(a). Now, a new dataset $Z^{L}$ can be collected and used in conjunction with the nominal dataset $Z^{N}$, the known $M$ and $z_{g}$, to estimate the change in mass $m$ and $\mathrm{CM} z_{m}$ and the desired properties can be found through (47).

\subsection{The Indirect Model}

Let us now look at how the indirect model for (48) can be formulated. Let $\vartheta^{T}=\left[I_{x}, d, k, m, z_{m}\right]$ be unknown parameters. The transfer function from the unknown input $u_{I}=a_{y}$ to the angular velocity $\dot{\phi}$ is then

$$
G_{1}(\mathrm{p}, \vartheta)=\frac{b_{0}(\vartheta) \mathrm{p}}{\mathrm{p}^{2}+a_{1}(\vartheta) \mathrm{p}+a_{2}(\vartheta)}
$$

where $b_{0}(\vartheta)=\left(M z_{g}+m z_{m}\right) / I_{1}(\vartheta), a_{1}(\vartheta)=d / I_{1}(\vartheta)$ and $a_{2}(\vartheta)=\left(k+M g z_{g}+m g z_{m}\right) / I_{1}(\vartheta)$.

In this example, there are no known or directly measured inputs, and only the signals $u_{I}=a_{y}, y_{I}=y_{2}$ and $y_{O}=y_{1}$ are used. This gives us the submodel

$$
y_{O}=G_{1}(\mathrm{p}, \vartheta) u_{I}+\frac{1}{b_{0}} G_{1}(\mathrm{p}, \vartheta) \tau=G_{O I}(\mathrm{p}, \vartheta) u_{I}+H_{O}(\mathrm{p}, \vartheta) \tau
$$

and the indirect input measurement

$$
y_{I}=\left(G_{1}(\mathrm{p}, \vartheta) \frac{z_{s} \mathrm{p}^{2}+g}{\mathrm{p}}-1\right) u_{I}+\frac{1}{b_{0}} G_{1}(\mathrm{p}, \vartheta) \frac{z_{s} \mathrm{p}^{2}+g}{\mathrm{p}} \tau=G_{I I}(\mathrm{p}, \vartheta) u_{I}+H_{I}(\mathrm{p}, \vartheta) \tau
$$

which results in the indirect model

$$
\tilde{G}_{O}(\mathrm{p}, \vartheta)=\tilde{G}_{O I}(\mathrm{p}, \vartheta)=G_{O I}(\mathrm{p}, \vartheta) G_{I I}^{-1}(\mathrm{p}, \vartheta)=\frac{\beta_{0}(\vartheta) \mathrm{p}}{\mathrm{p}^{2}+\alpha_{1}(\vartheta) \mathrm{p}+\alpha_{2}(\vartheta)}
$$

where $\beta_{0}(\vartheta)=-\left(M z_{g}+m z_{m}\right) / I_{2}(\vartheta), \alpha_{1}(\vartheta)=d / I_{2}(\vartheta), \alpha_{2}(\vartheta)=k / I_{2}(\vartheta)$ and $I_{2}(\vartheta)=I_{x}+$ $M z_{g}\left(z_{g}-z_{s}\right)+m z_{m}\left(z_{m}-z_{s}\right)$. Here, the angular velocity measurement $y_{2}$ was chosen as the output since this resulted in a proper indirect model. Moreover, note that the indirect model (52) is dependent on the same parameters as 48.

In this application, the continuous-time model (52) could be used directly to estimate the model parameters. However, the system has no difficult characteristics, for example, it is not stiff, and an approach based on a discrete-time model was chosen since it was deemed to give sufficient accuracy with low computational cost for the estimation problem. Here, the continuous-time model (52) was discretized using the bilinear transform $p=(2 q-2) /(T q+T)$ where $T$ is the sample period and $\mathbf{q}$ is the shift operator, i.e. $\mathbf{q} z_{t}=z_{t+1}$. The resulting discretetime model can be written as

$$
y_{O, t}^{k}=\left(\varphi_{t}^{k}\right)^{T} \theta_{k}(\vartheta)+\tilde{\tau}_{t}^{k}, \quad k=1,2
$$

where $k=1$ corresponds to the nominal dataset and $k=2$ corresponds to the new (loaded) dataset. The regression vector is given by $\varphi_{t}^{k}=\left[-y_{O, t-1}^{k},-y_{O, t-2}^{k}, y_{I, t}^{k}-y_{I, t-2}^{k}\right]^{T}$. The parameter 
vectors are given by

$$
\theta_{2}^{T}(\vartheta)=\left[\left(2 \alpha_{2}(\vartheta) T^{2}-8\right) / \bar{I}_{2}(\vartheta),\left(4-2 \alpha_{1}(\vartheta) T+\alpha_{2}(\vartheta) T^{2}\right) / \bar{I}_{2}(\vartheta),-\left(2 \beta_{1}(\vartheta) T\right) / \bar{I}_{2}(\vartheta)\right]
$$

and $\theta_{1}(\vartheta)=\left.\theta_{2}(\vartheta)\right|_{m, z_{m}=0}$ where $\bar{I}_{2}(\vartheta)=4+2 \alpha_{1}(\vartheta) T+\alpha_{2}(\vartheta) T^{2}$.

\subsection{Extended Instrumental Variable Method}

There are two terms contributing to the output of (53), one that contains information about the interesting input-output relation and the second which contains a contribution from disturbances. An instrumental variable (IV) method is a correlation based method and uses instruments $\zeta_{t}^{k}$ to extract the interesting information, for example, the parameters $\vartheta$, from the data. A good instrument should be correlated with the regressors but be uncorrelated with the process disturbance, sensor biases and measurement noises. The extended IV estimator is given by

$$
\hat{\vartheta}=\underset{\vartheta}{\operatorname{argmin}} \sum_{k=1}^{n_{O}}\left\|Y^{k}-\Phi^{k} \theta_{k}(\vartheta)\right\|^{2}
$$

where $\|x\|^{2}=x^{T} x$. The data and regression matrices are

$$
Y^{k}=\frac{1}{N_{k}}\left[\begin{array}{lll}
\bar{\zeta}_{1}^{k} & \ldots & \bar{\zeta}_{N_{k}}^{k}
\end{array}\right]\left[\begin{array}{c}
\bar{y}_{O, 1}^{k} \\
\vdots \\
\bar{y}_{O, N_{k}}^{k}
\end{array}\right], \quad \text { and } \quad \Phi^{k}=\frac{1}{N_{k}}\left[\begin{array}{lll}
\bar{\zeta}_{1}^{k} & \ldots & \bar{\zeta}_{N_{k}}^{k}
\end{array}\right]\left[\begin{array}{c}
\left(\bar{\varphi}_{1}^{k}\right)^{T} \\
\vdots \\
\left(\bar{\varphi}_{N_{k}}^{k}\right)^{T}
\end{array}\right]
$$

where $\bar{\zeta}_{t}^{k}=L^{k}(\mathbf{q}) \zeta_{t}^{k}, \bar{y}_{O, t}^{k}=L^{k}(\mathbf{q}) y_{O, t}^{k},\left(\bar{\varphi}_{t}^{k}\right)^{T}=L^{k}(\mathbf{q})\left(\varphi_{t}^{k}\right)^{T}$ and $L^{k}(\mathbf{q}), \quad k=1, \ldots, n_{O}$, are stable pre-filters. For more details on the original extended IV method, see for instance, Söderström and Stoica (1989) or Ljung (1999).

One benefit of the estimator (55) is that it will be consistent under fairly general conditions. The challenge is to design the instruments $\zeta_{t}^{k}$ and the pre-filters $L^{k}(\mathrm{q}), k=1, \ldots, n_{O}$, such that the accuracy of the predictor is maximized. The approach used in this paper is the iterative approach introduced by Gilson, Garnier, Young, and Van den Hof (2011). During each iteration, the current estimate of the parameters is used to create the instruments $\bar{\zeta}_{t}^{k}$ and the pre-filters $L^{k}(\mathrm{q}), k=1, \ldots, n_{O}$, which are then used to get a new estimate of the parameters, and the iterations are terminated when the parameters have converged. The inand outputs are simulated with the current parameter estimate $\hat{\vartheta}$ by

$$
\left[\begin{array}{c}
\hat{y}_{O, t}^{k} \\
-\hat{y}_{I, t}^{k}
\end{array}\right]=\left[\begin{array}{c}
G_{O I}(\mathbf{q}, \hat{\vartheta}) \\
-\underline{G_{I I}}(\mathbf{q}, \hat{\vartheta})
\end{array}\right] \hat{F}_{\delta}(\mathbf{q}) \delta_{t}^{k}
$$

where $\hat{F}_{\delta}(\mathrm{q})$ is a discrete-time estimate of $F_{\delta}(\mathrm{p})$. Here, the $k^{\text {th }}$ instrument is

$$
\bar{\zeta}_{t}^{k}=L^{k}(\mathbf{q})\left[-\hat{y}_{O, t-1}^{k} \ldots-\hat{y}_{O, t-\hat{n}_{O}^{k}}^{k} \quad \hat{u}_{K, t-1}^{k} \ldots \hat{u}_{K, t-\hat{n}_{K}^{k}}^{k} \quad \hat{y}_{I, t-1}^{k} \ldots \hat{y}_{I, t-\hat{n}_{I}^{k}}^{k} \quad \hat{y}_{D, t-1}^{k} \ldots \hat{y}_{D, t-\hat{n}_{D}^{k}}^{k}\right],
$$

where the constants $\hat{n}_{i}^{k}, i=O, K, I, D$, are the number of time lags included in $\bar{\zeta}_{t}^{k}$. Here, $\hat{n}_{O}^{k}=0$ means that $\hat{y}_{O, t}^{k}$ is not included in $\bar{\zeta}_{t}^{k}$. The pre-filters $L^{k}(\mathrm{q}), k=1, \ldots, n_{O}$, are estimated from the residuals. For more details on how to choose instruments and pre-filters, see for example, Forssell and Chou (1998), Gilson and Van den Hof (2005) or Gilson et al. (2011). 


\subsection{Experimental Setup, Datasets and Estimator Settings}

Data was collected from the pendulum seen in Figure 6(b) and have been used previously in Linder, Enqvist, and Gustafsson (2014). The known physical quantities listed on the first row of Table 1 were carefully measured using a scale and caliper.

Figure 6(b) shows two Xsens MTi IMUs (orange). The IMU mounted on the pendulum was used to measure the tangential acceleration $a_{s}$ and the angular velocity $\dot{\phi}$ using the accelerometer and the gyro of the IMU, respectively. The reference signal $\delta$ was measured using the accelerometer of the IMU mounted on the cart. Note that the reference signal $\delta$ thus was measured with noise but that this only has an impact on the accuracy of the parameter estimates.

Two nominal datasets where the mass $M$ and $\mathrm{CM} z_{g}$ were known. In two datasets, there was an unknown additional mass at an unknown location. All datasets were collected at $50 \mathrm{~Hz}$ with 30000 samples in each dataset and they were created by manually dragging the cart seen in Figure 6(b) back and forth on the rail. A part of one dataset can be seen in Figure 8. One nominal and one additional mass dataset were used for estimation and the other two were used for validation.

Here, the residuals $\varepsilon_{t}^{k}=y_{O, t}^{k}-\left(\varphi_{t}^{k}\right)^{T} \theta_{k}(\vartheta), k=1,2$, were used to estimate the noise models

$$
\hat{H}^{k}\left(\mathbf{q}, \eta^{i}\right)=\frac{1+\bar{c}_{1}^{k} \mathbf{q}^{-1}+\bar{c}_{2}^{k} \mathbf{q}^{-2}+\bar{c}_{3}^{k} \mathbf{q}^{-3}}{1+\bar{\gamma}_{1}^{k} \mathbf{q}^{-1}+\bar{\gamma}_{2}^{k} \mathbf{q}^{-2}}, \quad k=1,2,
$$

which were then incorporated into the pre-filters

$$
L^{k}(\mathbf{q})=\frac{1+\bar{\gamma}_{1}^{k} \mathbf{q}^{-1}+\bar{\gamma}_{2}^{k} \mathbf{q}^{-2}}{\left(1+\bar{c}_{1}^{k} \mathbf{q}^{-1}+\bar{c}_{2}^{k} \mathbf{q}^{-2}+\bar{c}_{3}^{k} \mathbf{q}^{-3}\right)\left(1+\bar{\alpha}_{1}^{k} \mathbf{q}^{-1}+\bar{\alpha}_{2}^{k} \mathbf{q}^{-2}\right)}, \quad k=1,2,
$$

that were used in the iterative estimator. Note that $1+\bar{\alpha}_{1}^{k} \mathrm{q}^{-1}+\bar{\alpha}_{2}^{k} \mathrm{q}^{-2}, k=1,2$, were the denominator of (52) after discretization. These noise models might not be the optimal choice but gave sufficient performance in this application.
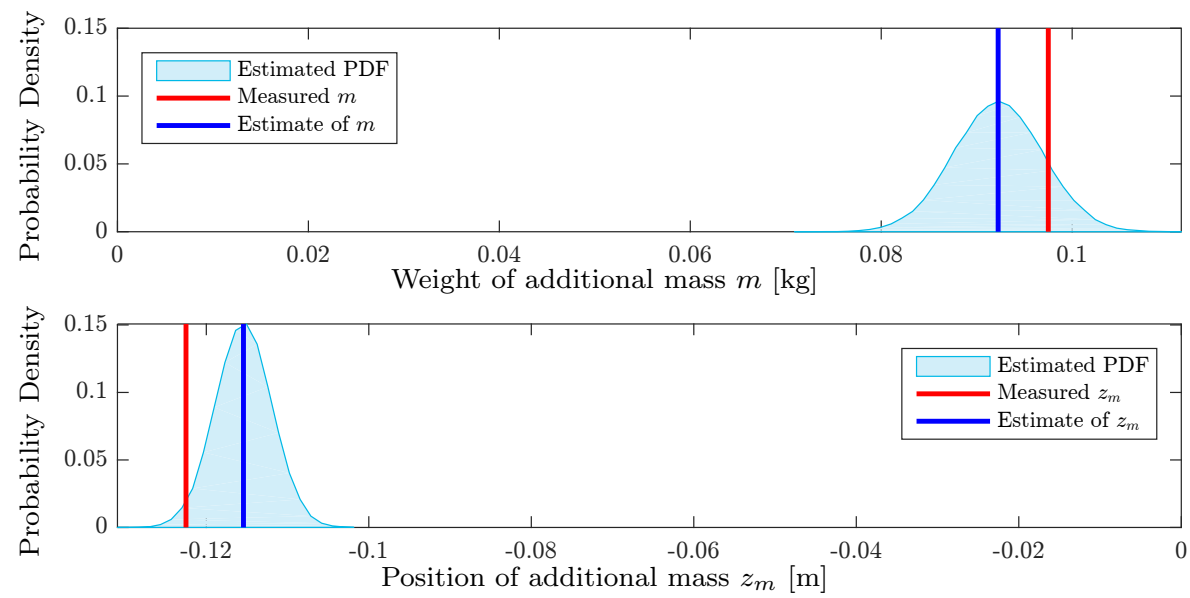

Figure 7. The result from the estimation of the parameters in Table 1 Here, only the parameters with known true values are shown. Red corresponds to the measured or calculated physical quantity in Table 1 Light blue is the estimated probability density function $\mathcal{N}\left(\hat{\vartheta}, \hat{P}_{\vartheta}\right)$ in the $m$ and $z_{m}$ directions and blue corresponds to the sample mean. Top: $m$ and bottom: $z_{m}$. 
Table 1. The measured physical quantities and the results from identification. The first row corresponds to the measured values. The second row contains the estimated values and the third row contains the relative errors.

\begin{tabular}{c|cc|ccccc|cc} 
& $M$ & $z_{g}$ & $I_{x}$ & $d$ & $k$ & $m$ & $z_{m}$ & $m z_{m}$ & $m z_{m}^{2}$ \\
\hline Measured & 1.324 & 0.0211 & - & - & - & 0.0980 & -0.123 & -0.0119 & 0.0015 \\
Estimated & - & - & 0.0071 & 0.0032 & 1.84 & 0.0922 & -0.115 & -0.0126 & 0.0012 \\
Relative error & - & - & - & - & - & $5.41 \%$ & $5.77 \%$ & $10.9 \%$ & $16.0 \%$
\end{tabular}

\subsection{Results}

The results of the IV estimator described in Section 5.3 can be seen in Figure 7 and Table 1. Figure 7 shows the estimated probability density functions created using $\vartheta$ and the asymptotic covariance matrix $\hat{P}_{\vartheta}$ where it was assumed that the estimates were normally distributed. The blue bars correspond to the mean and the red bars correspond to the physical quantities in the first row of Table 1 . Note that the true values of $m$ and $z_{m}$ are within three standard deviations of the estimated value. Furthermore, the estimated value of the moment of inertia was less than $1 \%$ larger than a value that has been calculated based on measurements of the dimensions of weights of the major components and mechanics theory. Smaller components, such as screws, or complicated soft components, such as the cables, were neglected and the calculated moment of inertia was thus probably smaller than the true inertia.

In addition to comparing the physical quantities, the model was validated by simulating (50) and (51) with the estimated parameters using the measured cart acceleration $\delta$ as input and $\tau=0$. The fit to the nominal data was $75.4 \%$ for (50) and $75.8 \%$ for (51). Similarly, the fit to the loaded data was $73.8 \%$ for $(50)$ and $74.3 \%$ for (51). Here, the fit is defined as the normalized root mean square error given by Tangirala (2014, p. 364). Figure 8 shows 24 typical seconds of the loaded dataset together with the simulated output from (50). The model captures the period of the signal well, but both under- and overestimates the peaks. This could be due to the disturbance in the measured input $\delta$ or due to nonlinearities in the restoring and friction torques of the real system.

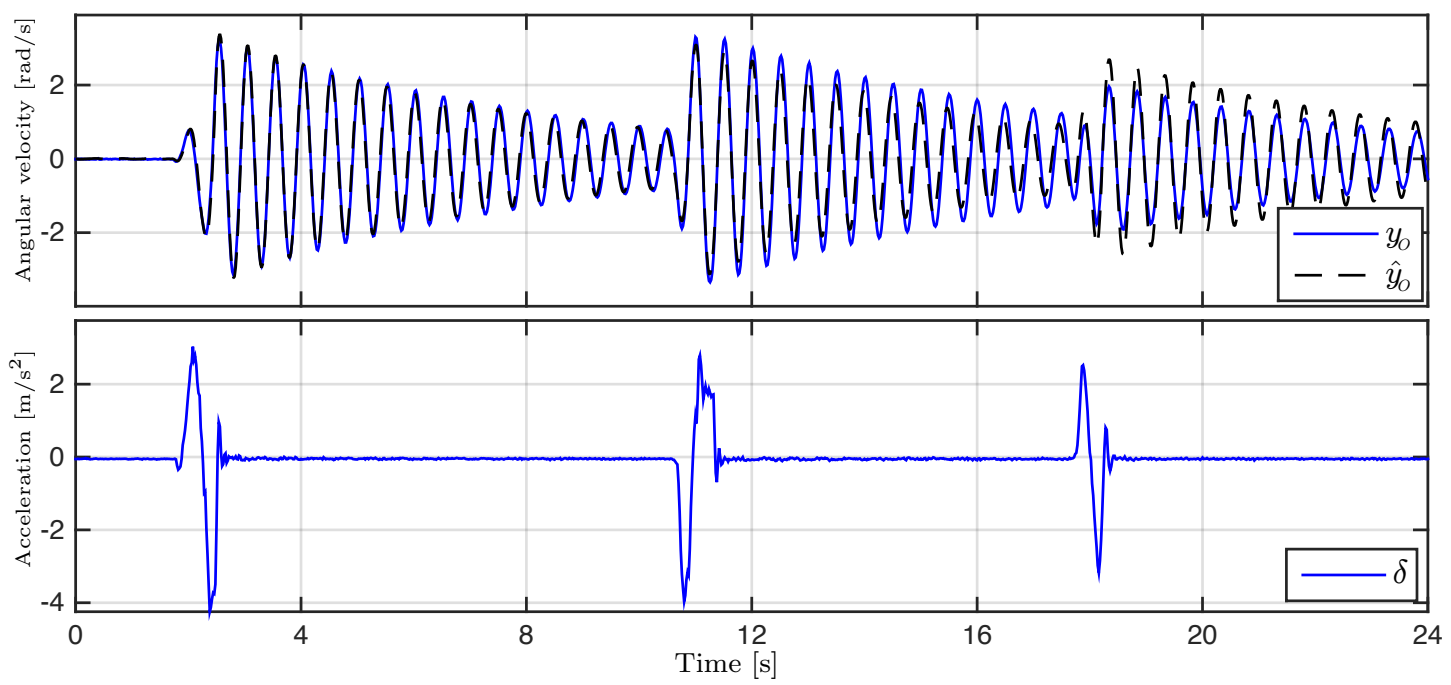

Figure 8. A typical part of the validation data with additional mass (blue). The simulated model output (dashed black) captures the periods of the data well but has troubles with both over- and underestimation of the peaks. 


\section{Conclusions}

In this paper, the recently proposed framework of Linder and Enqvist (2015) has been extended to handle additional input measurements and a deeper analysis has been given. In this framework, the indirect model (12), where the unknown input has been substituted with direct and indirect input measurements, has been proposed to obtain an estimate of the desired model (1). A discussion about the properties of the indirect model and the predictor in- and output selection has been given. It has been shown that due to the generality of the indirect modeling framework it unifies a number of already existing system identification problems that are contained as special cases of the indirect model (12). Furthermore, benefits of using the indirect framework have been discussed in the context of identifiability, consistency and variance properties.

Several methods can be used to estimate the indirect model (12) and in the experimental verification section, an iterative instrumental variable method has been suggested as one example. In addition to pointing to a recent application of the indirect framework in Linder et al. (2015a) and (2015b), the proposed framework and estimation method have been applied to data from a real pendulum experiment with good results.

\section{References}

Abed-Meraim, K., Qiu, W., \& Hua, Y. (1997, Aug). Blind system identification. Proceedings of the IEEE, 85(8), 1310-1322. doi: 10.1109/5.622507

Aljanaideh, K., Ali, A., Holzel, M., Kukreja, S., \& Bernstein, D. (2012). Sensor-to-sensor identification of hammerstein systems. In Proceedings of the 51st IEEE conference on decision 8 control (p. 28462851). Maui, Hawaii, USA. doi: 10.1109/CDC.2012.6426385

Anguelova, M. (2007). Observability and identifiability of nonlinear systems with applications in biology (PhD thesis). Chalmers University of Technology, Gothenburg, Sweden.

Bazanella, A. S., Gevers, M., \& Miškovic, L. (2010). Closed-loop identification of MIMO systems: A new look at identifiability and experiment design. European Journal of Control, 16(3), 228-239.

Bellman, R., \& Åström, K. (1970). On structural identifiability. Mathematical Biosciences, 7(3-4), 329 339. doi: 10.1016/0025-5564(70)90132-X

D’Amato, A. M., Brzezinski, A. J., Holzel, M. S., Ni, J., \& Bernstein, D. S. (2009, July). Sensor-only noncausal blind identification of pseudo transfer functions. In Proceedings of the 15th IFAC symposium on system identification (pp. 1698-1703). Saint-Malo, France.

Dankers, A. (2014). System identification in dynamic networks (PhD thesis). Delft University of Technology, The Netherlands.

Diop, S., \& Fliess, M. (1991). Nonlinear observability, identifiability, and persistent trajectories. In Proceedings of the 30th IEEE conference on decision and control. Brighton, England. doi: 10.1109/CDC.1991.261405

Forssell, U., \& Chou, C. (1998). Efficiency of prediction error and instrumental variable methods for closedloop identification. In Proceedings of the 37th IEEE conference on decision and control (pp. 1287-1288 vol.2). Tampa, Florida, USA.

Forssell, U., \& Ljung, L. (1999). Closed-loop identification revisited. Automatica, 35(7), 1215 - 1241. doi: 10.1016/S0005-1098(99)00022-9

Garnier, H., \& Wang, L. (2008). Identification of continuous-time models from sampled data. Springer.

Garnier, H., \& Young, P. C. (2014). The advantages of directly identifying continuous-time transfer function models in practical applications. International Journal of Control, 87(7), 1319-1338. doi: 10.1080/00207179.2013.840053

Gilson, M., Garnier, H., Young, P. C., \& Van den Hof, P. M. (2011). Optimal instrumental variable method for closed-loop identification. IET Control Theory \& Applications, 5(10), 1147-1154. 
Gilson, M., \& Van den Hof, P. (2005). Instrumental variable methods for closed-loop system identification. Automatica, 41(2), 241-249.

Hermann, R., \& Krener, A. (1977). Nonlinear controllability and observability. IEEE Transactions on Automatic Control, 22(5), 728-740.

Hou, M., \& Müller, P. (1994, Jun). Disturbance decoupled observer design: a unified viewpoint. Automatic Control, IEEE Transactions on, 39(6), 1338-1341. doi: 10.1109/9.293209

Hou, M., \& Patton, R. (1998, Mar). Optimal filtering for systems with unknown inputs. Automatic Control, IEEE Transactions on, 43(3), 445-449. doi: 10.1109/9.661621

Jung, Y., \& Enqvist, M. (2013). Estimating models of inverse systems. In Proceedings of the 52nd IEEE conference on decision \& control. Firenze, Italy. doi: 10.1109/CDC.2013.6761022

Lancaster, P., \& Tismenetsky, M. (1985). The theory of matrices: With applications. Academic Press.

Linder, J., \& Enqvist, M. (2015, October). On Indirect Input Measurements. In Proceedings of the 17th IFAC symposium on system identification. Beijing, China.

Linder, J., Enqvist, M., Fossen, T. I., Johansen, T. A., \& Gustafsson, F. (2015a, August). Modeling for IMU-based Online Estimation of Ship's Mass and Center of Mass. In Proceedings of the 10th IFAC Conference on Manoeuvring and Control of Marine Craft (MCMC). Copenhagen, Denmark.

Linder, J., Enqvist, M., Fossen, T. I., Johansen, T. A., \& Gustafsson, F. (2015b, August). Online Estimation of Ship's Mass and Center of Mass Using Inertial Measurements. In Proceedings of the 10th IFAC Conference on Manoeuvring and Control of Marine Craft (MCMC). Copenhagen, Denmark.

Linder, J., Enqvist, M., \& Gustafsson, F. (2014, December). A closed-loop instrumental variable approach to mass and center of mass estimation using IMU data. In Proceedings of the 53rd IEEE conference on decision 8 control. Los Angeles, CA, USA.

Linder, J., Enqvist, M., Gustafsson, F., \& Sjöberg, J. (2014, August). Identifiability of physical parameters in systems with limited sensors. In Proceedings of the 19th IFAC world congress. Cape Town, South Africa.

Ljung, L. (1999). System identification: Theory for the user (2nd edition). Prentice Hall.

Ljung, L., \& Glad, T. (1994). On global identifiability for arbitrary model parametrizations. Automatica, 30(2), 265 - 276. doi: 10.1016/0005-1098(94)90029-9

Mahata, K., \& Garnier, H. (2006). Identification of continuous-time errors-in-variables models. Automatica, 42(9), 1477 - 1490. doi: 10.1016/j.automatica.2006.04.012

Shumway, R., \& Stoffer, D. (2010). Time Series Analysis and Its Applications: With R Examples. Springer.

Söderström, T. (2007). Errors-in-variables methods in system identification. Automatica, 43(6), 939 - 958. doi: $10.1016 /$ j.automatica.2006.11.025

Söderström, T., \& Stoica, P. (1989). System identification. Prentice Hall.

Söderström, T., Wang, L., Pintelon, R., \& Schoukens, J. (2013). Can errors-in-variables systems be identified from closed-loop experiments? Automatica, 49(2), 681-684.

Stockham, T. G., Cannon, T. M., \& Ingebretsen, R. B. (1975, April). Blind deconvolution through digital signal processing. Proceedings of the IEEE, 63(4), 678-692. doi: 10.1109/PROC.1975.9800

Tangirala, A. (2014). Principles of system identification: Theory and practice. CRC Press.

Van den Hof, P., Dankers, A., Heuberger, P., \& Bombois, X. (2013). Identification of dynamic models in complex networks with prediction error methods, basic methods for consistent module estimates. Automatica, 49(10), 2994 - 3006. doi: 10.1016/j.automatica.2013.07.011

Walter, É. (1982). Identifiability of state space models: With applications to transformation systems. Springer-Verlag Berlin. 